\title{
CONSTRUÇÃO DE WEBLOG COMO FERRAMENTA PARA 0 ENSINO DO USO RACIONAL DE MEDICAMENTOS NO CURSO DE FARMÁCIA ${ }^{1}$
}

\author{
WEBLOG CONSTRUCTION IN PHARMACY \\ COURSE AS A TOOL FOR TEACHING
}

RATIONAL USE OF MEDICINES

Joyce Costa Melgaço Faria ${ }^{1}$, Daniela Garcia Rezende Junqueira ${ }^{1}$, Raissa Carolina Fonseca Cândido ${ }^{1}$, Cristiane Aparecida Menezes de Pádua ${ }^{1}$, Alba Valéria Souto Melo

Moraes $^{1}$, Alessandra Caroline Montes Frade ${ }^{1}$, Daniela Fernandes Silva ${ }^{1}$, Érika Paz de Souza Santos ${ }^{1}$, Louise Cristina

Oliveira Santos ${ }^{1}$, Mariana Fontoura Lana Nascimento ${ }^{1}$, Weverton Teixeira da Silva ${ }^{1}$, Alessandra Rezende Mesquita ${ }^{1}$, Edson Perini ${ }^{1}$

\section{RESUMO}

A informação correta é determinante na qualidade do uso de medicamentos e na tomada de decisões em saúde. Assim, é fundamental a formação de profissionais capazes de interpretar a evidência científica sobre medicamentos, transformando-a em informação de fácil acesso. Nesse sentido, o Centro de

${ }^{1}$ Universidade

Federal de Minas

Gerais (UFMG), Belo

Horizonte, Brasil.

\footnotetext{
${ }^{1}$ Uma versão anterior deste texto foi apresentada e publicada nos anais do I Congresso de Inovação e Metodologias de Ensino, realizado em Belo Horizonte, em 14 e 15 de outubro de 2015, pela Diretoria de Inovação e Metodologias de Ensino da Universidade Federal de Minas Gerais (GIZ-UFMG).
} 
Estudos do Medicamento (CEMED) da Faculdade de Farmácia da Universidade Federal de Minas Gerais (UFMG) promove a formação voltada para o uso racional de medicamentos, utilizando a divulgação de informações por meio de weblog (Blog do CEMED). A publicação no weblog depende do papel ativo dos discentes, os quais devem identificar as fontes de informação, selecionar os temas e elaborar, corrigir e divulgar as matérias. O blog tem registrado um movimento intenso de consulta, impulsionado pela interatividade das redes sociais. A iniciativa demonstra a evidente demanda por informações na área e se apresenta como importante recurso pedagógico, favorecendo o desenvolvimento de competências como avaliação crítica da literatura, comunicação escrita e trabalho em equipe.

Palavras-chave: Weblog. Farmácia. Uso racional de medicamentos.

\section{INTRODUÇÃO}

$\mathrm{Na}$ atualidade, os medicamentos são parte importante do sistema de atenção à saúde, sendo a tecnologia sanitária mais empregada no processo de cuidado (ACÚRCIO, 2003; BRASIL, 2012). No entanto, o aumento da morbimortalidade relacionada aos medicamentos tem sido reconhecido como fator de risco para a saúde (LLIMÓS; FAUS, 2003). Evidências comprovam que o consumo de medicamentos fora dos padrões da racionalidade científica gera doenças, prolonga internações, dificulta a relação profissional-usuário e promove aumento de 
custos (PERINI, 2003; PERES; JOB, 2010; MOYNIHAN; HENRY, 2006).

A superação dessa dinâmica comprovadamente danosa ao usuário e aos sistemas de saúde está associada, dentre outras medidas, à produção e à veiculação de informações corretas e acessíveis sobre o uso racional de medicamentos para a sociedade (WHO, 2004). Estudos demonstram que as informações sobre medicamentos divulgadas pela mídia e pela internet representam hoje uma das principais formas de educação em saúde para usuários e profissionais (MORETTI; OLIVEIRA; SILVA, 2012; BARRETO et al., 2013). Em pesquisa realizada por Moretti, Oliveira e Silva (2012), 90\% dos entrevistados relataram já ter buscado informações sobre a própria saúde na internet e $79 \%$ afirmaram que já realizaram pesquisas sobre a saúde de seus familiares. Além disso, $80 \%$ dessa população afirma considerar a internet uma das principais fontes de informação em saúde.

Nessa perspectiva, a produção de informações é fundamental para influenciar decisões em saúde que promovam benefício com mínimo risco de danos aos usuários, inclusive no que diz respeito ao uso racional de medicamentos. No cenário internacional, a produção de informação de qualidade e isenta de vieses comerciais é ferramenta essencial para facilitar e estimular a implantação de políticas públicas fundamentadas em pesquisas científicas (SURE, 2011). Ademais, é fundamental a formação de profissionais capazes de interpretar a evidência científica sobre medicamentos, transformando-a em informação de fácil acesso aos demais profissionais de saúde e aos usuários. 
Considerando essa influência das informações sobre medicamentos nas ações de profissionais e usuários, novas estratégias de comunicação estão sendo utilizadas para oferecer essa informação em formato acessível e inteligível, com conteúdo balanceado e responsável. O uso de ferramentas digitais, como weblogs, tanto para veiculação de informações em saúde como para o desenvolvimento de recursos humanos, tem sido relatado na literatura (BOULOS; MARAMBA; WHEELER, 2006; CAIN; DILLON, 2010; THIELST, 2007).

Nesse sentido, o Centro de Estudos do Medicamento (CEMED) da Faculdade de Farmácia da Universidade Federal de Minas Gerais (FAFAR-UFMG) tem utilizado a tecnologia de weblog para o desenvolvimento de novas práticas no ensino de graduação, promovendo a integração entre ensino, extensão e pesquisa. O weblog é utilizado como instrumento para capacitar o discente a transmitir informações científicas sobre o uso racional de medicamentos em linguagem acessível para um público heterogêneo, seja ele profissional de saúde, seja ele leigo. Nosso objetivo é descrever a experiência de construção de weblog como ferramenta de ensino no curso de Farmácia.

\section{DESCRIÇÃO}

\section{Histórico}

O trabalho do CEMED teve início em 2009, com a produção de informação científica sobre medicamentos e a divulgação ativa dessa iniciativa ao público por meio de um boletim 
impresso em edições mensais, nomeado Boletim Atrás da Estante. O boletim ficava exposto na biblioteca da FAFARUFMG, em um espaço criado especialmente para isso (Espaço Calvin, em alusão ao personagem dos quadrinhos Calvin e Haroldo), pensado para ser um ambiente de leitura e convívio. Nesse local, onde foram colocadas duas estantes expositoras de revistas, poltronas e mesa de centro, o CEMED divulgava, sempre em matérias de rápida leitura, uma coletânea de informações compiladas de diferentes fontes (periódicos científicos e sites de organizações idôneas), após análise do conteúdo, da relevância e da atualidade. Os discentes protagonizavam o trabalho, assumindo responsabilidades desde a decisão sobre os temas a serem abordados e a busca da literatura pertinente até a elaboração e a divulgação das matérias. Cópias das fontes utilizadas (artigos, capítulos ou matérias em sites) ficavam expostas nesse espaço, junto a jornais diários e revistas de notícias.

Em 2011, por iniciativa dos estudantes do CEMED, foram criados um perfil institucional do centro e uma fanpage no Facebook@, para dar mais ampla divulgação ao boletim. Em fevereiro de 2012, essa experiência levou à criação de um weblog (Blog do CEMED - http://cemedmg.wordpress.com), inicialmente com o objetivo de oferecer mais facilidade e comodidade para o acesso aos textos produzidos para a comunidade da Faculdade de Farmácia. Em 2014 foi criado também um perfil no Twitter@C. Atualmente, a fanpage no Facebook@ é utilizada para divulgar os textos produzidos para o weblog, como também as notícias relacionadas ao Sistema Único de Saúde (SUS) (principalmente deliberações e notas do Ministério da Saúde e da Agência Nacional de Vigilância 
Sanitária, a ANVISA), alertas da Organização Mundial da Saúde (OMS) e de agências reguladoras de medicamentos de outros países, como Food and Drug Administration (FDA) e European Medicines Agency (EMEA).

Aliadas a essas inciativas, outras estratégias de ensino utilizadas são: a oferta de uma disciplina optativa para o curso de graduação em Farmácia (FASO20 - Tópicos em Informação sobre Medicamentos) e a realização dos Seminários do CEMED. $\mathrm{Na}$ disciplina, os professores buscam aprofundar teoricamente a discussão sobre a importância da informação científica adequada na qualificação do consumo de medicamentos e atuar na construção de habilidades com a produção prática de informações para um grupo maior de alunos. Os Seminários do CEMED consistem em palestras expositivas, abertas a toda a comunidade, sobre temas gerados pelo weblog ou assuntos afins com vistas à busca de mais segurança e qualidade no consumo de medicamentos.

\section{Processo de trabalho para publicação no weblog}

O processo de publicação no weblog depende do papel ativo dos discentes na construção da informação, eles devem identificar temas de interesse e elaborar as matérias para publicação. Nesse sentido, o processo de trabalho pode ser dividido em cinco etapas, que serão descritas a seguir.

- Determinação do tema para publicação: a partir de consultas sistemáticas e periódicas a uma lista de fontes de informação sobre medicamentos, os discentes selecionam 
temas de interesse para publicação no blog. As fontes trazem evidência científica atual e de qualidade, e a lista tem característica dinâmica, pois inclusões e exclusões de fontes de informação podem ser realizadas se houver consenso do grupo.

- Reunião de editoria: nessa etapa, são definidas as matérias a serem publicadas e as ações necessárias para publicação, são distribuídas as tarefas aos discentes e estabelecidos os prazos. A reunião inicia-se com a exposição pelo discente do resultado da busca de temas nas fontes de informação. Os temas são discutidos em relação ao escopo e à relevância, sendo selecionados aqueles relativos à eficácia e à segurança na utilização de medicamentos, à educação farmacêutica e às práticas em saúde, principalmente no que concerne ao SUS. Selecionados os temas de interesse, é definido o estudante responsável pela elaboração da matéria, a qual poderá ser um texto para o blog ou postagens informativas mais enxutas para leitura nas redes sociais (Facebook@ e Twitter( )), sempre com explicitação das fontes de consulta e formas de acesso.

- Elaboração da matéria para o blog: com base na busca e na interpretação crítica da literatura científica em saúde, o discente deve redigir um texto de tamanho aproximado de uma 
lauda e com linguagem acessível ao público leigo ou profissional de saúde, caso se trate respectivamente de um texto informativo ao consumidor ou um texto técnico.

- Revisão: nessa etapa, o texto elaborado é enviado, por meio eletrônico, aos demais membros do grupo, os quais deverão avaliálo criticamente em um prazo estabelecido. As correções podem ser de cunho estrutural (questionamentos gramaticais ou adequação da linguagem) ou técnico (que diz respeito ao conteúdo da informação a ser veiculada aos leitores). Assim, são realizadas rodadas sucessivas de correção, em que o autor deve produzir nova versão do texto, acatando ou não as sugestões. Esse processo é realizado até que o texto esteja adequado e todos apoiem a publicação do material.

- Publicação: terminada a revisão, cabe ao autor publicar o próprio texto no weblog e divulgálo nas redes sociais, o que é feito por meio do compartilhamento de uma mensagem, em linguagem adequada e atrativa aos leitores. É também responsabilidade do autor responder aos comentários realizados sobre os textos que escreveu, bem como às dúvidas que possam ser encaminhadas pelos leitores, seja no weblog, nas redes sociais ou por e-mail. 
Vale ressaltar que todo o processo é supervisionado por dois docentes coordenadores do grupo, que programam as discussões e orientam atividades desenvolvidas com base no conhecimento e na experiência que possuem.

\section{RESULTADOS E DISCUSSÃO}

Conforme descrito por Silva (2008):

Os blogs educacionais são espaços eletrônicos individuais ou coletivos para se partilhar informações, ideias, opiniões, materiais e referências. Um espaço destinado à leitura e produção de pequenos textos que podem ser comunicados, questionados e comentados por outros leitores (SILVA, 2008, p. 3).

Essas ferramentas permitem que os membros da equipe possam compartilhar conhecimentos de forma simples e rápida, além de possibilitar a interação e a reflexão crítica (SAUER et al., 2005; BOULOS; MARAMBA; WHEELER, 2006). Nos últimos anos, pode ser observada na literatura uma quantidade substancial de blogs gerados por prestadores de cuidados de saúde, o que demonstra os benefícios potenciais dessas ferramentas na transmissão de informação em saúde (THIELST, 2007; SANTORO, 2007; SETHI, 2007). No estudo realizado por Cain e Dillon (2010), foram identificados 44 blogs na área de farmácia designados para a transmissão de informações em saúde/medicamentos.

O CEMED tem desenvolvido uma rica experiência ao se apropriar do alcance das ferramentas digitais atualmente disponíveis para disseminar informações com embasamento científico e isentas de vieses comerciais. Desde o início do 
weblog do CEMED, participaram da experiência dezoito discentes (bolsistas e voluntários) do curso de graduação em Farmácia da UFMG. A equipe é atualmente composta por dois docentes, uma pesquisadora afiliada (voluntária), duas farmacêuticas e sete estudantes de graduação em Farmácia da UFMG. A permanência mínima dos discentes no grupo foi de um ano, chegando alguns à permanência por um período de três a quatro anos.

A proposta inicial, relativamente simples, do Boletim Atrás da Estante, de levar informações sobre medicamentos para a comunidade da Faculdade de Farmácia da UFMG ensejou o ingresso do CEMED no ambiente digital, e o Blog do CEMED acabou se transformando em um veículo com intenso movimento de consulta e visibilidade. Até o final de dezembro de 2015, foram publicados no Blog do CEMED 196 textos. Em conjunto, os textos alcançaram mais de 250 mil acessos, o Gráfico 1 mostra o número crescente de acessos por ano.

Gráfico 1 - Acessos ao Blog do CEMED por ano

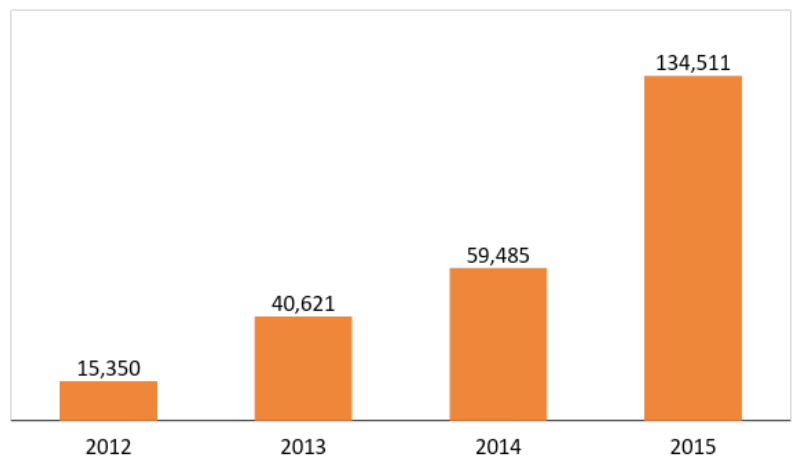

Fonte: Elaborado pelos autores, 2016. 
As visualizações foram originadas de 120 países dos cinco continentes. Vinte e quatro países apresentaram mais de cem acessos, sendo a maioria proveniente do Brasil (230.238), Estados Unidos (11.012), Portugal (5.484), Argentina (436) e Espanha (365). O alcance das publicações tem aumentado: a média de acessos por texto é de aproximadamente 1.305, e, até o final de dezembro de 2015, contabilizamos 28 textos publicados com mais de mil acessos, oito desses totalizando mais de 5 mil acessos e dois com mais de 30 mil acessos. A Tabela 1 apresenta as dez matérias mais acessadas do blog.

Tabela 1 - Matérias mais acessadas do Blog do CEMED

\begin{tabular}{lc}
\hline \multicolumn{1}{c}{ Textos } & Número de acessos \\
\hline $\begin{array}{l}\text { Omeprazol e outros inibidores } \\
\text { da bomba de prótons: } \\
\text { consequências do uso } \\
\text { prolongado }\end{array}$ & 38.613 \\
\hline $\begin{array}{l}\text { Medicamentos para diarreia: } \\
\text { entender as causas para fazer }\end{array}$ & 30.429 \\
$\begin{array}{l}\text { uma boa escolha } \\
\text { Fosfoetanolamina: realidade, } \\
\text { promessa ou mais um } \\
\text { pesadelo? }\end{array}$ & 18.664 \\
\hline $\begin{array}{l}\text { Como tomar corretamente } \\
\text { os anti-inflamatórios não } \\
\text { esteroides }\end{array}$ & 15.516 \\
\hline $\begin{array}{l}\text { Medicamentos e álcool, uma } \\
\text { combinação que merece } \\
\text { atenção }\end{array}$ & 12.555 \\
\hline
\end{tabular}

(continua) 


\begin{tabular}{lc}
\hline \multicolumn{1}{c}{ Textos } & Números de acessos \\
\hline $\begin{array}{l}\text { Medicamento de referência, } \\
\text { genérico, similar e a RDC № } \\
58 / 2014\end{array}$ & 6.868 \\
\hline $\begin{array}{l}\text { Paracetamol e o risco de } \\
\text { alterações hepáticas: uma dose } \\
\text { a mais de informação }\end{array}$ & 6.251 \\
\hline $\begin{array}{l}\text { Saúde mental: clonazepam é o } \\
\text { psicotrópico mais consumido } \\
\text { no Brasil }\end{array}$ & 6.188 \\
\hline $\begin{array}{l}\text { Metotrexato: medicamento } \\
\text { potencialmente perigoso }\end{array}$ & 2.772 \\
\hline $\begin{array}{l}\text { Varfarina: o que é importante } \\
\text { saber para garantir que os } \\
\text { benefícios superem os riscos }\end{array}$ & 2.767 \\
\hline
\end{tabular}

Fonte: Elaborado pelos autores, 2016.

A divulgação de informações em saúde no weblog e nas redes sociais implica o reconhecimento da utilidade e da importância dessas ferramentas pela influência que exercem no cuidado à saúde. Podem ser ofertadas aos usuários informações que vão desde o significado de sinais e sintomas das doenças até informações sobre os medicamentos que irão tratálos. Assim, é fundamental que esses espaços virtuais sejam ocupados com informações cientificamente embasadas e em linguagem acessível, fazendo frente à intensa veiculação de material de cunho mais comercial e propagandístico que informativo. Essa perspectiva é reforçada pela disseminação internacional da informação produzida pelo Blog do CEMED, apesar de as matérias serem publicadas em língua portuguesa. Fato que nos leva a acreditar que o idioma, embora fator 
limitante, não tem sido empecilho para a busca de informação qualificada. Adicionalmente, o elevado número de acessos oriundos do Brasil demonstra êxito na oferta de informação sobre medicamentos aos profissionais de saúde e usuários brasileiros.

Desde a criação, o weblog obteve 327 comentários, entre mensagens recebidas e respostas a essas mensagens. Essa interatividade permitida pelo weblog, e ampliada pelas redes sociais pelas trocas de mensagens, comentários e curtidas sobre as postagens, reforça a importância do uso dessas mídias no fomento de discussões sobre temas relacionados à saúde, principalmente no que concerne ao uso racional dos medicamentos.

O acesso dos visitantes ao Blog do CEMED é direcionado principalmente pelo Facebook (C) (mais de 76 mil) e a partir de mecanismos de busca, como Google Search, Bing.com, Ask. com e Search Babylon (mais de 100 mil). Esses mecanismos de acesso, seja pelas redes sociais seja por ferramentas de busca, demonstram que a procura por informações sobre saúde na internet é uma realidade, corroborando com outros estudos na literatura (MORETTI; OLIVEIRA; SILVA, 2012; BARRETO et al., 2013), e reitera a efetividade das redes sociais para disseminar informações de qualidade em saúde.

No que se refere à questão pedagógica, a utilização do weblog tem o intuito de promover o aprimoramento da formação dos discentes. Na atualidade, estudantes vivem em uma sociedade imediatista, dinâmica e apoiada em tecnologia, na qual há aumento explosivo não só da quantidade total de informação, mas também na facilidade do acesso (FRENK et 
al., 2010; ABENFARBIO, 2013). Assim, a utilização de um meio no qual a atual geração mostra um entusiasmo espontâneo, como os weblogs, é uma boa estratégia para se respeitar a linguagem cotidiana dos discentes e convidá-los a explorar novas possibilidades dentro do próprio universo de expressão (FONSECA, 2012).

Outro aspecto a ser considerado no cenário atual é que o aprendizado estanque não é suficiente para atender as demandas da sociedade, portanto é necessária uma mudança nos modos de aprender e ensinar. Assim, o discente deve ser protagonista no processo de aprendizagem, devendo aprender a aprender. Nesse ponto, a utilização do weblog do CEMED permitiu a participação ativa e responsável dos estudantes em todo o processo de produção da informação, iniciando-se com a seleção do tema até a divulgação dos conteúdos.

Os resultados do processo de aprendizagem dos discentes por meio do weblog podem ser observados no desenvolvimento dos estudantes nas competências para interpretação e avaliação crítica da literatura científica e produção dos textos em linguagem inteligível ao público. Isso corrobora com a literatura que enfatiza a necessidade de mudanças nas estratégias utilizadas no ensino farmacêutico, com mais ênfase na aquisição das competências para a prática profissional (BRANDT, 2000; OMS, 2001; FIP, 2008; AWAISU et al., 2010; HIGH LEVEL GROUP ON THE MODERNIZATION OF HIGHER EDUCATION, 2013). Esses documentos sugerem diversas competências desejadas ao discente farmacêutico egresso, das quais, destacam-se: capacidade de pensar criticamente, 
tomada de decisão, habilidades de comunicação, resolução de problemas e capacidade de trabalhar de forma colaborativa.

Durante a construção dos textos para o Blog do CEMED, o primeiro momento desafia o aluno à compreensão de critérios importantes para a seleção de fontes de consulta e o estabelecimento de parâmetros de avaliação da qualidade da informação a ser utilizada como referência. Ao elaborar o texto, o aluno aprimora habilidades de escrita e de comunicação científica em linguagem acessível, além de desenvolver experiência de trabalho em equipe. Nas discussões, os estudantes são estimulados a defender pontos de vista, buscando sempre respaldo científico para tanto, assim como a assimilar sugestões e críticas para o enriquecimento do trabalho realizado. Posteriormente, a divulgação dos textos nas redes sociais estimula o desenvolvimento da capacidade de síntese e criatividade, pois o estudante precisa resumir a ideia mais importante do texto, utilizando abordagem que desperte interesse no leitor, numa linguagem acessível e adequada à dinâmica dessas redes. Além disso, a atividade proporciona a aprendizagem de conteúdos específicos, em diversas áreas de conhecimento, como: farmacoepidemiologia, farmacoterapia e saúde baseada em evidências, por meio das pesquisas em fontes de informação.

Em relação aos Seminários do CEMED, no período de maio de 2011 a dezembro de 2015, foram realizadas 26 edições, sendo a média de público de 34 pessoas por seminário e o maior público alcançado de 92 espectadores. Alguns temas de destaque foram: "Epidemia de ebola", "Canabinoides como novas perspectivas de tratamento das epilepsias" e "Prevenção 
de erros de medicação em idosos". Esses seminários têm permitido o aprofundamento das discussões e proporcionado à comunidade, principalmente aos graduandos, o contato com experiências e visões diferenciadas. Além disso, o contato com profissionais de áreas de atuação diversas também contribui para a ampliação das bases conceituais dos participantes.

A disciplina Tópicos em Informação sobre Medicamentos, por sua vez, foi ofertada em três semestres e cursada por aproximadamente quarenta estudantes, incluindo discentes em diferentes estágios de formação. Textos selecionados do Blog do CEMED são utilizados como material didático para ilustrar uma das formas de veiculação da "informação ativa", em que a decisão da comunicação da informação é uma iniciativa do informante, e não uma demanda direta do solicitante (leigo ou profissional de saúde). Nessa atividade, o aluno exercita a habilidade da comunicação escrita, utilizando linguagem apropriada e acessível para diferentes públicos.

A oferta dessa disciplina tem ampliado o acesso à experiência vivida no CEMED para um grupo maior de estudantes. Embora não seja possível reproduzir toda a experiência, ela cumpre o papel de levar aos discentes as bases fundamentais do processo de busca e acesso à informação e a importância desse processo na sociedade. Além disso, para os discentes em períodos iniciais do curso de Farmácia, a disciplina representa uma das primeiras oportunidades de discussão sobre a importância da provisão de informação para o uso racional de medicamentos, bem como sobre a necessidade da formação de profissional de saúde com esse perfil. 
Mais investigações são necessárias, para que se possa determinar com mais detalhes o impacto do uso desse recurso tecnológico na aprendizagem e na satisfação dos discentes. Desse modo, a experiência servirá de subsídio para novas pesquisas sobre ensino farmacêutico que serão realizadas pelos integrantes do CEMED.

\section{CONSIDERAÇÕES FINAIS}

O acesso facilitado à internet e o predomínio de atividades que envolvem o uso do computador reforçam o potencial dessas ferramentas tanto no ensino de farmácia quanto no atendimento à demanda da sociedade por informação sobre o uso de medicamentos. A ampliação do volume de acessos ao Blog do CEMED é indicativo da relevância e da aceitabilidade das informações transmitidas e do alcance do público a ser atendido pelos discentes na futura vivência deles como profissionais de saúde.

No processo de trabalho do CEMED, o aprimoramento da formação dos discentes dá-se na identificação, na compreensão crítica da literatura científica e na produção de informações seguras e isentas de conflitos de interesses, possibilitando uma formação profissional diferenciada, que valoriza o impacto que a informação e a educação em saúde têm no uso racional de medicamentos. A qualidade dos textos publicados no Blog do CEMED demonstra a rica experiência extraclasse vivenciada pelos estudantes e a oportunidade de trabalhar com ferramentas virtuais trouxe para eles um enfoque relativamente novo, que pode ser modelo para outras áreas de ensino na Farmácia. 


\section{REFERÊNCIAS}

ACÚRCIO, F. A. (Org.). Medicamentos e assistência farmacêutica. Belo Horizonte: Coopmed Editora Médica, 2003. p. 9-30.

ASSOCIAÇÃO BRASILEIRA DE ENSINO FARMACÊUTICO E BIOQUÍMICO. ABENFARBIO. Metodologias ativas: aplicações e vivências em educação farmacêutica. 2. ed. Brasília: Abenfarbio, 2013. 160 p.

AWAISU, A. et al. Malaysian pharmacy students' assessment of an Objective Structured Clinical Examination (OSCE). Am J Pharm Educ, v. 74, n. 2, Article 34, 2010.

BARRETO, N. M. P. B. et al. YouTube ${ }^{\circledR}$ pode oferecer educação de qualidade?. In: 30 Congresso de Cardiologia da SOCERJ. Revista Brasileira de Cardiologia, Rio de Janeiro, v. 26, n. A, p. 66-68, abr. 2013.

BOULOS, M. N.; MARAMBA, I.; WHEELER, S. Wikis, blogs and podcasts: a new generation of Web-based tools for virtual collaborative clinical practice and education. $B M C$ Med Educ. v. 6, p. 41, 2006.

BRANDT, B. F. Effective teaching and learning strategies. Pharmacother, v. 20, n. 10 Pt 2, p. 307-316, 2000.

BRASIL. Ministério da Saúde. Secretaria de Ciência, Tecnologia e Insumos Estratégicos. Uso racional de medicamentos: temas selecionados/Ministério da Saúde, Secretaria de Ciência, Tecnologia e Insumos Estratégicos - Brasília: Ministério da Saúde, 2012. 156 p. 
CAIN, J.; DILLON, G. Analysis of pharmacy-centric blogs: types, discourse themes, and issues. J Am Pharm Assoc. v. 50, n. 6, p. 714-719, 2010.

FONSECA, A. A. Portfólio digital: o blog no recurso pedagógico no ensino superior. Semina: Ciências Sociais e Humanas. v. 33, n. 1, p: 81-90, 2012.

FRENK, J. et al. Health professionals for a new century: transforming education to strengthen health systems in an interdependent world. The Lancet, v. 376, p. 19231958, 2010.

HIGH LEVEL GROUP ON THE MODERNIZATION OF HIGHER EDUCATION. Report to the European Commission on improving the quality of teaching and learning in Europe's higher education institutions. Luxembourg: Publications Office of the European Union. 2013.

INTERNATIONAL PHARMACEUTICAL FEDERATION. FIP. A global framework for quality assurance of pharmacy education. version 1. Netherlands: FIP. 2008. 29 p.

LLIMÓS, F. F.; FAUS, M. J. Importance of medicine-related problems as risk factors. The Lancet, v. 362, p. 123-129, 2003.

MORETTI, F. A.; OLIVEIRA, V. E.; SILVA, E. M. K. Acesso a informações de saúde na internet: uma questão de saúde pública? Revista da Associação Médica Brasileira, São Paulo, v. 58, n. 6, p. 650-658, nov./dez. 2012.

MOYNIHAN, R.; HENRY, D. The Fight against Disease Mongering: Generating knowledge for action. PLoSMed, v. 3, n. 4, e191, p. 1-4, abr. 2006. 
OMS. Organização Mundial da Saúde. Guia do instrutor em práticas da boa prescrição médica. Genebra: OMS, 2001. $103 \mathrm{p}$.

PERES, G.; JOB, J. R. P. P. Indústria farmacêutica: percepções éticas de estudantes de Medicina. Revista Brasileira de Educação Médica, v. 34, n. 4, p. 515-524, 2010.

PERINI, E. Assistência farmacêutica: fundamentos teóricos e conceituais. In: ACURCIO, F. A. (Org.). Medicamentos e assistência farmacêutica. Belo Horizonte: Coopmed Editora Médica, 2003. p. 9-30.

SANTORO, E. Podcasts, wikis and blogs: the web 2.0 tools for medical and health education. Recent Prog Med. v. 98, n. 10, p. 484-494, 2007.

SAUER, I. M. et al. "Blogs" and "Wikis" are valuable software tools for communication within research groups. Artif Organs. v. 29, p. 82. 2005.

SETHI, S. K. Blog/web log - a new easy and interactive website building tool for a non-net savvy radiologist. $J$ Thorac Imaging. v. 22, n. 2, p. 115-119, 2007.

SILVA, A. Blog educacional: o uso das novas tecnologias no ensino, 2008. Disponível em: http://intranet.ufsj.edu. br/rep_sysweb/File/vertentes/Vertentes_31/adriana_ da_silva.pdf.. Acesso em: jan. 2016.

THE SURE COLLABORATION. SURE Guides for preparing and using evidence-based policy briefs. Version 2.1 [updated November 2011]. The SURE Collaboration, 2011. Disponível em: http://global.evipnet.org/SUREGuides/. Acesso em: 26 jun. 2015. 
THIELST, C. B. Weblogs: a communication tool. J Healthc Manag. v. 52, n. 5, p. 287-289, 2007.

WORLD HEALTH ORGANIZATION. WHO. Pharmacovigilance: ensuring the safe use of medicines. WHO Policy Perspectives on Medicines, Geneva, n. 9, p. 1-6, out. 2004.

\begin{abstract}
Accurate information is determinant to the quality use of medication and decision making in health. Thus, training professionals to interpret scientific evidence on medicines and turn that into easy-access information is fundamental. In this sense, the Centro de Estudos do Medicamento (CEMED) from the School of Pharmacy - Federal University of Minas Gerais (UFMG) promotes formation focusing on the rational use of medicines disseminating information through the Blog do CEMED. Content publication in the weblog relies on the active role of students, who identify sources of information, select topics, prepare, check and post them. The Blog has registered intense consultation access, driven by social networks interactivity. The initiative demonstrates clear need for information in this area and presents as an important pedagogical resource, promoting the development of competencies such as critical evaluation of literature, written communication and teamwork.
\end{abstract}

Keywords: Weblog. Pharmacy. Rational use of medicines. 


\section{Joyce Costa Melgaço Faria}

Farmacêutica graduada pela Universidade Federal de Minas Gerais (UFMG). Atualmente é mestranda do Programa de Pós-Graduação em Medicamentos e Assistência Farmacêutica e vinculada ao Centro de Estudos do Medicamento (Departamento de Farmácia Social, Faculdade de Farmácia da UFMG).

joycecmfaria@hotmail.com

\section{Daniela Garcia Rezende Junqueira}

Atuou como editora associada ao Centro de Estudos do Medicamento (CEMED/UFMG; 2007-2015) e atualmente é diretora-fundadora da editora Evidências em Saúde, Cocoordenadora do Cochrane Adverse Effects Methods Group, consultora científica da empresa canadense Medlior e pesquisadora afiliada à University of Sydney. Formada em Farmácia (2007), mestre e doutora em Ciências Farmacêuticas (2008; 2012) e pós-doutora em Ciências da Reabilitação (2014). danijunqueira@gmail.com

\section{Raissa Carolina Fonseca Cândido}

Farmacêutica graduada pela Universidade Federal de Minas Gerais (UFMG). Atualmente é colaboradora do Centro de Estudos do Medicamento (Departamento de Farmácia Social, Faculdade de Farmácia da UFMG) e do Instituto para Práticas Seguras no Uso de Medicamentos (ISMP Brasil). raissa.karol@gmail.com

\section{Cristiane Aparecida Menezes de Pádua}

Farmacêutica, mestre e doutora em Saúde Pública. É professora do Departamento de Farmácia Social da Faculdade de Farmácia da Universidade Federal de Minas Gerais (UFMG) e professora 
colaboradora do Centro de Estudos do Medicamento (CEMED) da UFMG.

campadua@farmacia.ufmg.br

\section{Alba Valéria Souto Melo Moraes}

Farmacêutica graduada pela Universidade Federal de Minas Gerais (UFMG). Possui mestrado profissional em Administração pela Fundação Pedro Leopoldo. Atuou como farmacêutica e colaboradora do Centro de Estudos do Medicamento (Departamento de Farmácia Social, Faculdade de Farmácia da UFMG).

albasouto@uol.com.br

\section{Alessandra Caroline Montes Frade}

Graduanda em Farmácia pela Universidade Federal de Minas Gerais (UFMG). Atualmente é colaboradora do Centro de Estudos do Medicamento (Departamento de Farmácia Social, Faculdade de Farmácia da UFMG) e do Laboratório de Farmacognosia (Departamento de Produtos Farmacêuticos da Faculdade de Farmácia da UFMG).

alecmfrade@gmail.com

\section{Daniela Fernandes Silva}

Graduanda em Farmácia pela Universidade Federal de Minas Gerais (UFMG). Atualmente é colaboradora do Centro de Estudos do Medicamento (Departamento de Farmácia Social, Faculdade de Farmácia da UFMG).

danielafernandessilva@yahoo.com.br

\section{Érika Paz de Souza Santos}

Graduada em Farmácia pela Universidade Federal de Minas Gerais (UFMG). Atualmente é colaboradora do Centro de 
Estudos do Medicamento (Departamento de Farmácia Social, Faculdade de Farmácia da UFMG).

eryka009@hotmail.com

\section{Louise Cristina Oliveira Santos}

Graduanda em Farmácia pela Universidade Federal de Minas Gerais (UFMG). Atualmente é colaboradora do Centro de Estudos do Medicamento (Departamento de Farmácia Social, Faculdade de Farmácia da UFMG).

louiseoliveira42@gmail.com

\section{Mariana Fontoura Lana Nascimento}

Graduanda em Farmácia pela Universidade Federal de Minas Gerais (UFMG). Atualmente é colaboradora do Centro de Estudos do Medicamento (Departamento de Farmácia Social, Faculdade de Farmácia da UFMG).

maryana_fin@hotmail.com

\section{Weverton Teixeira da Silva}

Graduando em Farmácia pela Universidade Federal de Minas Gerais (UFMG). Atualmente é colaborador do Centro de Estudos do Medicamento (Departamento de Farmácia Social, Faculdade de Farmácia da UFMG).

weverton.ts@gmail.com

\section{Alessandra Rezende Mesquita}

Graduada em Farmácia, mestre em Ciências Farmacêuticas e doutora em Ciências da Saúde pela Universidade Federal de Sergipe. Professora no Departamento de Farmácia Social da Faculdade de Farmácia da Universidade Federal de Minas Gerais (UFMG). Atualmente é colaboradora do Centro de 
Estudos do Medicamento (Departamento de Farmácia Social, Faculdade de Farmácia da UFMG).

alessandra_pharmacia@hotmail.com

\section{Edson Perini}

Farmacêutico, mestre em Parasitologia e doutor em Ciência Animal pela Universidade Federal de Minas Gerais (UFMG). Atualmente é professor e coordenador do Centro de Estudos do Medicamento (Departamento de Farmácia Social, Faculdade de Farmácia da UFMG).

edsonperini@gmail.com 
